\title{
Intelligent Automatic V6 and V8 Engine Sound Detection Based on Artificial Neural Network
}

\author{
Wenny Vincent $^{1}$, Astuti Winda ${ }^{2, *}$, and Mahmud Iwan Solihin ${ }^{3}$ \\ ${ }^{1}$ Automotive and Robotics Engineering Department, BINUS ASO School of Engineering, Bina \\ Nusantara University, Alam Sutera Boulevard no. 1, Alam Sutera-Serpong, Tangerang-Banten 15325, \\ Indonesia \\ ${ }^{2}$ Computer Engineering Department, BINUS ASO School of Engineering, Bina Nusantara University, \\ Jl. K. H. Syahdan No. 9, Kemanggisan, Palmerah, Jakarta 11480, Indonesia \\ ${ }^{3}$ Faculty of Engineering and Built Enviroment, UCSI University, No. 1, Jalan Menara Gading, UCSI \\ Heights (Taman Connaught), Cheras 56000 Kuala Lumpur, Malaysia
}

\begin{abstract}
The sound of V6 or V8 engines has its own cultural appeal that cannot be replaced by the modern four-cylinder naturally aspirated or turbocharged engines. The identification of the type of engine by the sound is not an easy task, even for the professionals. An intelligent system that can identify V6 to V8 engines from various cars will give an insight of the features in the engine sounds that characterized the two different engines. In this work, an Artificial Neural Network (ANN) approach is applied for identifying cylinder of the engine based on the engine sound identification is proposed. The recorded sound of the engine is then processed in order to get some features which later be used in the proposed system. The Fast Fourir Transform (FFT) is adopted as a feature and later used as input to the Artificial Neural Network (ANN) based identifier. The Experimental results confirm the effectiveness of the proposed intelligent automatic six cylinder and eight cylinder engine based on Fast Fourier Transform (FFT) and Artificial Neural Network (ANN), since it resulting the training and testing accuracy of $100 \%$ and $100 \%$, respectively.
\end{abstract}

Key words: Automatics petrol identification, fast fourier transform, support vector machine.

\section{Introduction}

Nowadays, the development of the industrial revolution 4.0 in the autonomous vehicle sector is experiencing rapid growth. The advance autonomous technology involve many aspect in the vehicle, such as autonomous self driving, and full autonomous self driving [1]. One of the importance aspect in advance autonomous vehicle is engine mainatance. The engine maintainace system plays an important rule in vehicle system, since the engine is the main part in the automotive. Despite the common automotive trend toward smaller displacement engines to cut down emission and fuel consumption, there are still some market segments for high performance engine such as luxury, heavy-duty vehicles, and the "petrohead" communities. Especially for the last one, the sound of V6 or V8 engines has its

\footnotetext{
${ }^{*}$ Corresponding author: wastuti@binus.edu
} 
own cultural appeal that cannot be replaced by the modern four-cylinder naturally aspirated or turbocharged engines.

The identification of the type of engine by the sound is not an easy task, even for the professionals. An intelligent system that can identify V6 to V8 engines from various cars will give an insight of the features in the engine sounds that characterized the two different engines. Moreover, it can provide references for engine sound features that draw the interest of some certain "petrol-head"communities.

Feature extraction and intelligent system are the most main stages in this work . the sound signal is processing in the feature extraction method the feature data is the used as input to the intelligent system. Mel-frequency cepstral coefficient (MFCC) [2], linear predictive coding (LPC) [3], and Fast Frourier Transform (FFT) [4] are some of the technique to extract the feature. There are many intelligent system that often applied in the system such as support vertor machine [5], multi-layers neural network [2,6] and fuzzy logic [7].

In this work, an Artificial Neural Network (ANN) approach is applied for identifying cylinder of the engine based on the engine sound identification is proposed. The recorded sound of the engine is then processed in order to get some features which later be used in the proposed system. The Fast Fourier Transform (FFT) is adopted as a feature and later used as input to the Artificial Neural Network (ANN) based identifier.

\section{Literature review}

This section presents, the basic general process consist of two important parts, namely sound acquisition, feature extraction and pattern matching. Sound acquisition process to record the image by using a microphone. The next process of the recorded sound will be extracted by feature extraction. In this process, the extracted data is the use as input to the engine sound identification session. Finally, the sound engine recognition to make the training process to recognize the type of engine of the automobile.

\subsection{Fast Fourier Transform (FFT)}

Fast Fourier Transform (FFT), is the faster Algorithm of the Discrete Fourier Transform (DFT). Discrete Fourier transform (DFT) is a specific form of the Fourier Transform applied to a time wave, typically a sound. Each signal is arranged from a sine / cosine function which has a specified frequency and a relative amplitude [8].

Feature extraction process based on Fast Fourier Transform (FFT) [8], is extracted the signal in time domain convert into frequency domain [9]. FFT is given by Nithya et al. [10], as shown in Equation 1. The fast fourier transform of $f(x)$ is the function of $F(\omega)$ is explained as equation below [11];

$$
F(\omega)=\int_{-\infty}^{\infty} f(x) e^{-i \omega x} d x
$$

Where $i=\sqrt{-1}$ and $e^{i \theta}=\cos \theta+\sin \theta$

\subsection{Artificial neural network}

Artificial Neural networks algorithm is used to simulate same or all the characteristic of the biological neurons that form the structural constituents of the brain. Neural network can be trained to perform a particular function by updating the value of connections (weight) 
between elements [12]. Commonly neural networks are trained, so that particular input leads to a specific target output. The Multi-layer perceptron, as shown in Figure 1 is consists of three layers of neural networks; input layer, hidden layer and output layer [8].

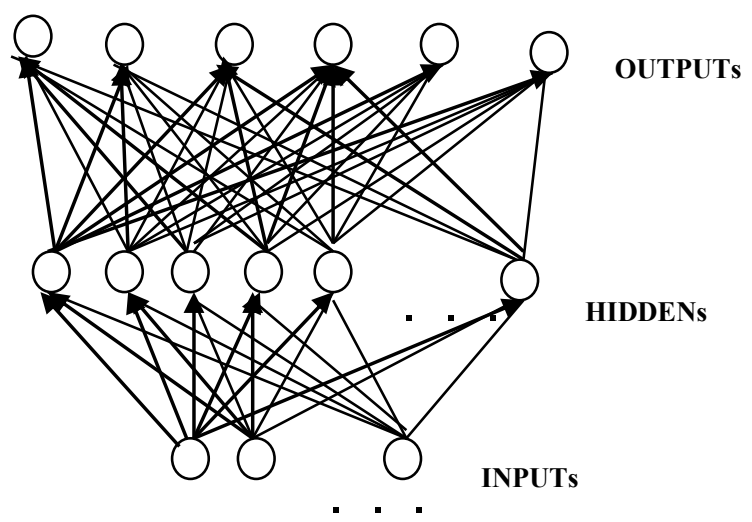

Fig. 1. MLP neural network.

\section{Proposed ANN-based engine sound identification system}

This paper used ANN as engine identification based on sound. A block diagram of conventional sound identification is shown in Figure 2. The system is trained to identify the engine type by each person speaking out a specific utterance into the microphone. The speech signal is digitized and some of them are carried out to create a template for the voice pattern and stored in the memory.

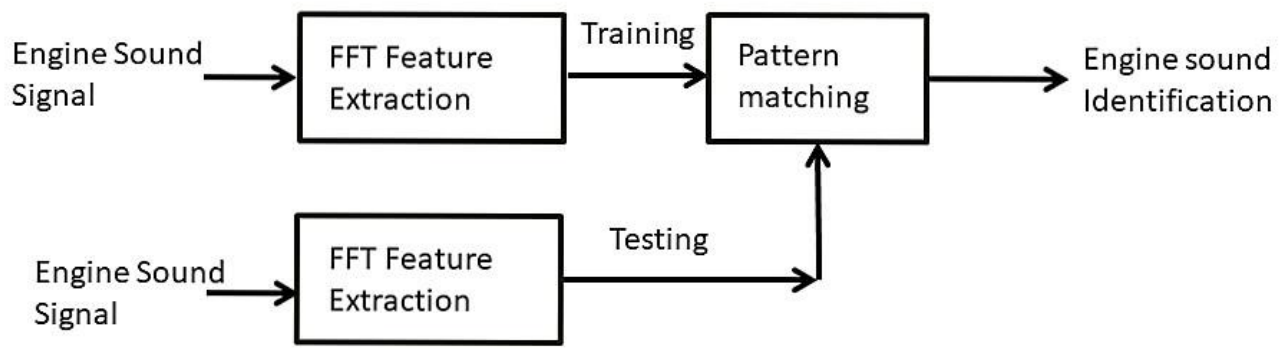

Fig. 2. Block diagram of conventional engine type identification system.

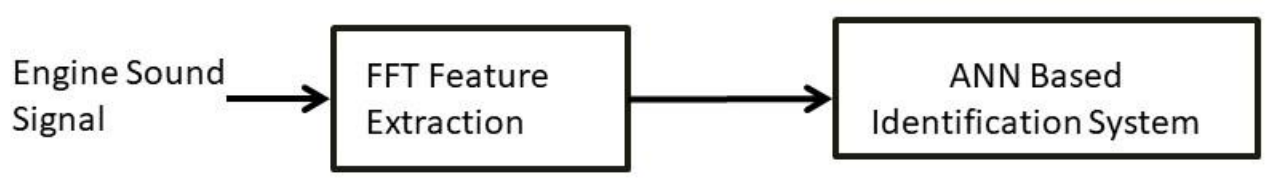

Fig. 3. Overall proposed engine type identification model

As shown in Figure 3, the sound engine is recorded by the sensor. The sound signal is then digitized in the computer. The digitaze signal is then extracted into the feature which used as input to the identification system. FFT technique is apply as extraction method which extract the engine signal into the numerical feature. ANN based method is applied as identification system which idenfity the engine data feature. 


\section{Experimental Work}

This section presents the performance of the proposed to automatic engine V6 and V8 engine sound identification based on FFT technique and machine learning approach. This section consists of three important subsections, they are experimental setup, experimental result and experimental analysis.

\subsection{Experimental Design}

The experimental design of engine type identification is shown in the Figure 4. The sound of the engine is recorded using a microphone. The records sound of the engine then sent to the PC thought 16 bits A/D with sampling frequency $44 \mathrm{KHz}$, all the signal is converted in the PC with processor 1.5 Mhz Pentium as show in the Table 1. The sound of the engine signal is recorded using microphone and then the recorded signal is sent to the computer through analog to digital (A/D) card.

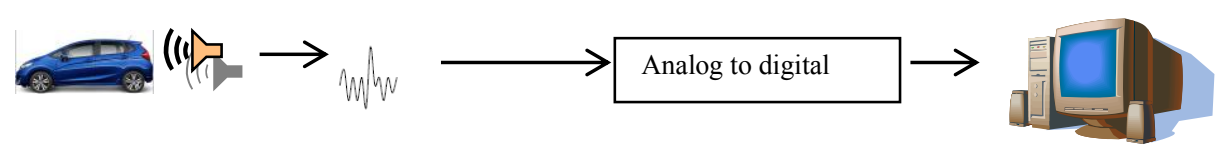

Fig. 4. Automatic V6 and V8 engine sound identification.

System identifies four different automotive engine sound from four different car with two types of V6 and V8 engine type, respectively.

Table 1. Specification of the system.

\begin{tabular}{|l|l|}
\hline Processor & $\mathbf{1 . 5} \mathbf{~ M H z}$, Intel \\
\hline A/D & 16 bits A/D \\
\hline Frequency sampling & $44 \mathrm{KHz}$ \\
\hline
\end{tabular}

\subsection{Experiment Result}

In order to evaluate the effectiveness of the proposed intelligent system on the cylinder engine identification system, the proposed system is installed. In this proposes system the specification microphones. The sound is recorded and captured. The raw recorded V8 engine signal and V6 engine as shown in Figure 5a and 5b, respectively.

Once the raw signal is recorded, then the FFT method is applied to the raw signal, as shown in Figure 6. The FFT result of the FFT on V6 and V8 engine sound signal. From eight sample peak amplitude from four different vehicles of each type, V6 and V8, respectively are obtained. Four different samples of each vehicle of different type of engine are used to train the system. Furthermore, the other four different samples of each of the vehicles samples are applied to test the system. In this experiment The ANN-based binaryclass classification is applied to perform the classification process of the training phases. The training classification rate for these two features based resulting $100 \%$ correct and the testing classification rate lies at $100 \%$. 


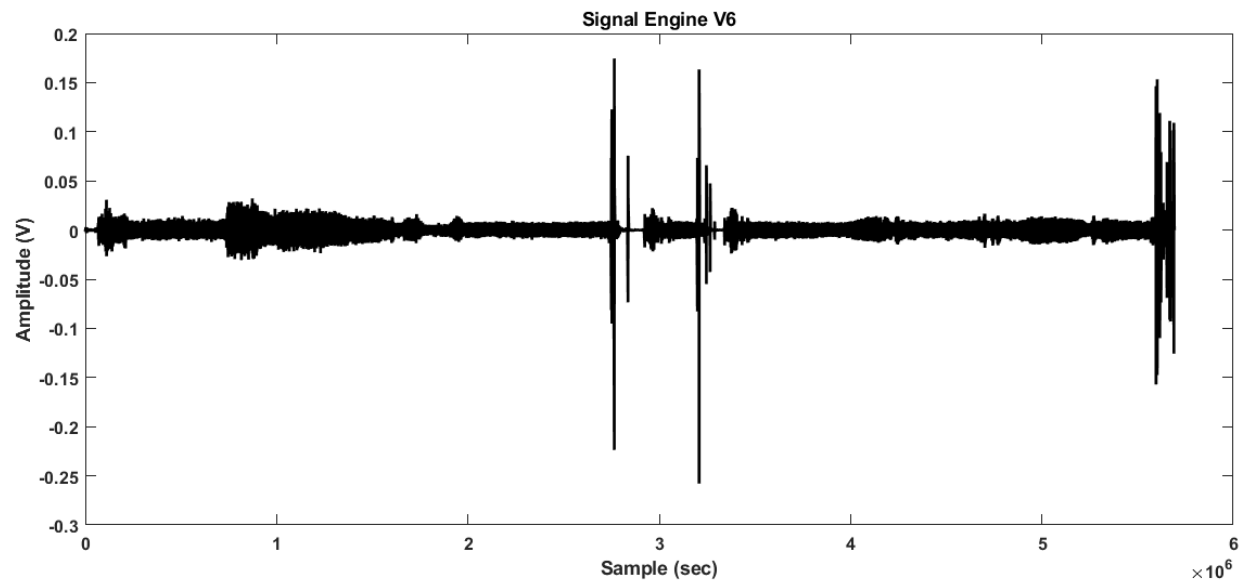

(a) Raw signal of V6 engine

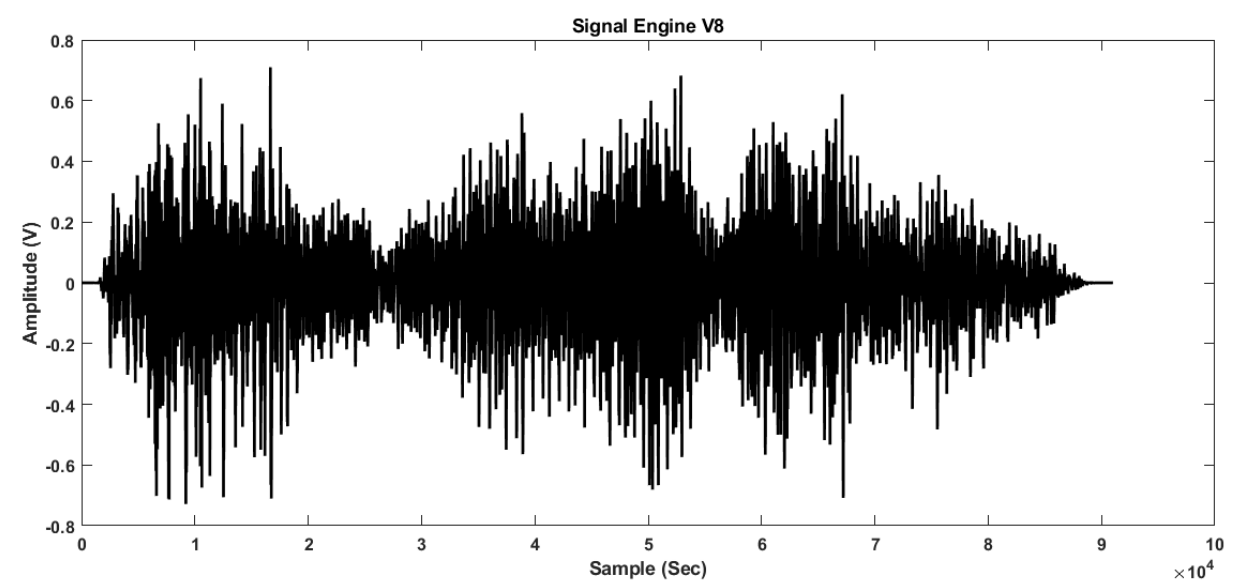

(b) Raw signal of V8 engine

Fig. 5. Raw V6 and V8 signal.

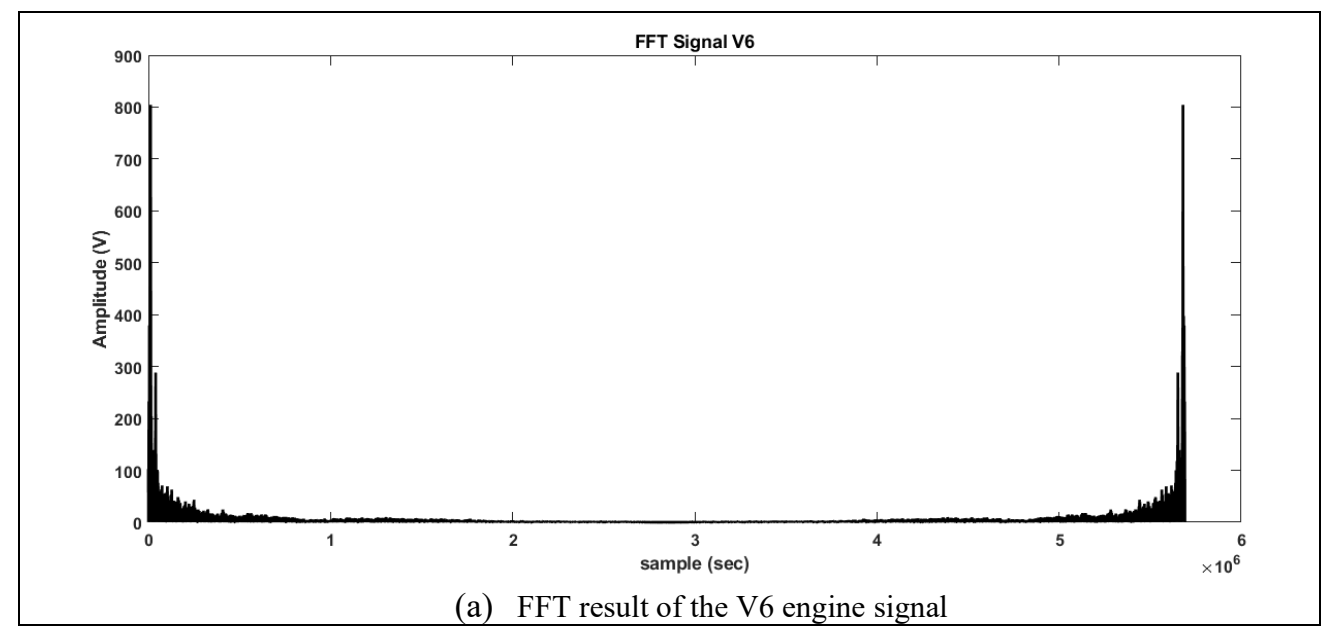




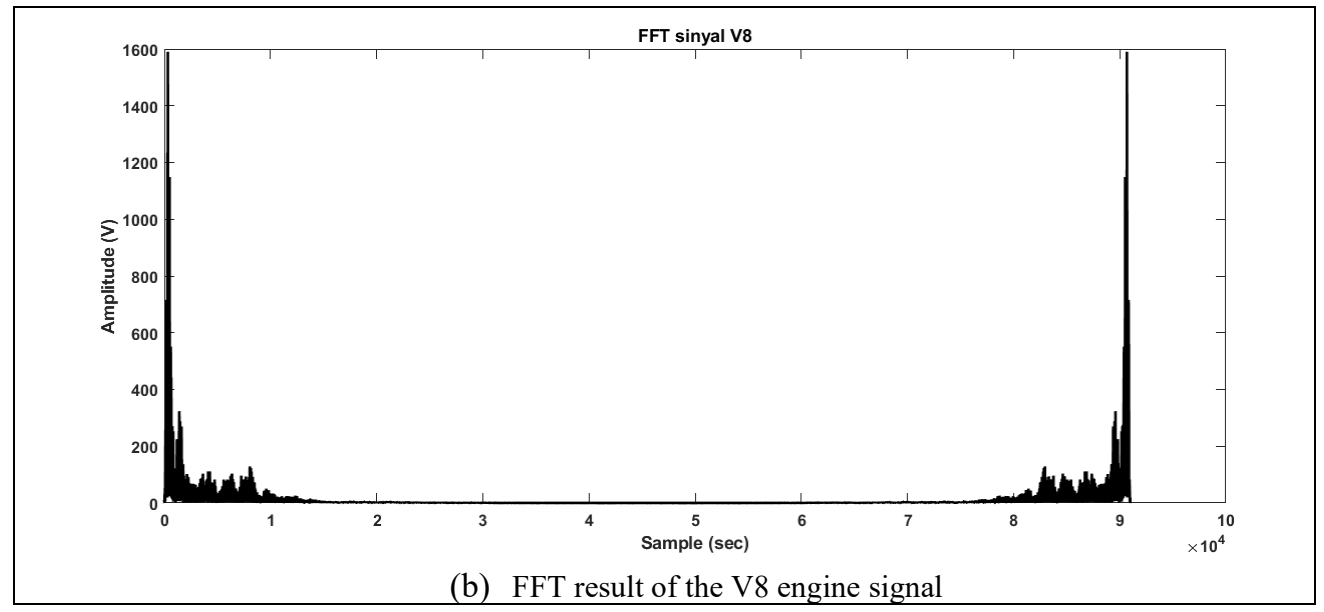

Fig. 6. FFT result of the V6 and V8 engines.

\subsection{Experiment analysis}

The distance between engine and microphone, noise environments and the stability of the sounds of the engine are affected with the recording quality. These factors impact the FFT algorithm. Table 2 discusses the accuracy between different types of V6 and V8 engine based on the sound of the engine. FFT algorithm feature extraction has applied as the feature extraction method. The result shows that the system has good accuracy, since it has $100 \%$ accuracy for both training and testing phases, respectively.

Table 2. Experimental results of training and testing phase.

\begin{tabular}{|l|c|c|}
\hline Type Classification & $\begin{array}{c}\text { Training } \\
\text { (Classification rate \%) }\end{array}$ & $\begin{array}{c}\text { Testing } \\
\text { (Classification rate \%) }\end{array}$ \\
\hline V6 engine type & 100 & 100 \\
\hline V8 engine type & 100 & 100 \\
\hline
\end{tabular}

\section{Conclusion}

The sound of V6 or V8 engines has its own cultural appeal that cannot be replaced by the modern four-cylinder naturally aspirated or turbocharged engines. The identification of the type of engine by the sound is not an easy task, even for the professionals. An intelligent system that can identify V6 to V8 engines from various cars will give an insight of the features in the engine sounds that characterized the two different engines.

In this work, an Artificial Neural Network (ANN) approach is applied for identifying cylinder of the engine based on the engine sound identification is proposed. The recorded sound of the engine is then processed in order to get some features which later be used in the proposed system. The Fast Fourier Transform (FFT) is adopted as a feature and later used as input to the Artificial Neural Network (ANN) based identifier. ANN-based engine sounds identification has been proposed and discuss in this paper. The significant FFT frequencies used as features. The result shows that FFT frequencies have successfully performed as good features. The accuracy of the ANN based engine sound identification using FFT frequencies is $100 \%$. 


\section{References}

1. Asean Development Bank. What does the Fourth Industrial Revolution mean for regional economic integration? Geneva: World Economic Forum (2017). https://www.adb.org/sites/default/files/publication/379401/asean-fourth-industrialrevolution-rci.pdf

2. N. Dave. Int. J. Adv. Res. Eng. Technol., 1,VI :1-5(2013). https://pdfs.semanticscholar.org/0b44/265790c6008622c0c3de2aa1aea3ca2e7762.pdf

3. Thiang, S. Wijoyo. Speech recognition using linear predictive coding and artificial neural network for controlling movement of mobile robot. International Conference on Information and Electronics Engineering (Bangkok, Thailand, 2011). IPCSIT, 6:179183(2011). http://www.ipcsit.com/vol6/36-E091.pdf

4. H. Ali, M.J.E. Salami, Wahyudi. Iris recognition system by using support vector machines. Proceedings of the International Conference on Computer and Communication Engineering 2008, ICCCE08: Global Links for Human Development (Kuala Lumpur, Malaysia, 2008). https://ieeexplore.ieee.org/document/4580657

5. T. Seehapoch, S. Wongthanavasu. Speech emotion recognition using support vector machines. The 5th International Conference on Knowledge and Smart Technology (KST), (Chonburi, Thailand, 2013). p. 86-91. https://ieeexplore.ieee.org/document/6512793

6. P. Potocnik. Neural networks: MATLAB examples. Slovenia: University of Ljubljana (2012).

https://www.academia.edu/22782673/Neural_Networks_MATLAB_examples_Neural_ Networks_course practical_examples_2012_Primoz_Potocnik

7. Wahyudi, W. Astuti, S. Mohamed. Journal of Computer Science, 3,5:274-280(2007). https://thescipub.com/abstract/10.3844/jcssp.2007.274.280

8. N.T. Deshpande, S. Ravishankar. Adv. Comput. Sci. Technol., 10,5:1173-1189(2017). https://pdfs.semanticscholar.org/c5cf/c1f5a430ad9c103b381d016adb4cba20ce4e.pdf

9. D. Gunawan, F.H. Juwono. Pengolahan Sinyal Digital dengan Pemograman Matlab. [Digital Signal Processing with Matlab Programming] Yogyakarta: Graha Ilmu (2012). [in Bahasa Indonesia]. https://grahailmu.id/product/pengolahan-sinyal-digital-denganpemrograman-matlab/

10. A. Nithya, C. Lakshmi, R. Krithiga (2017). IJPAM, 113,12: 57-66(2017). https://www.researchgate.net/publication/317040705_Iris_Recognition_Based_on_GL CM_and_FFT_Feature_Set_Fusion

11. J. Sueuer. A very short introduction to sound analysis for those who like elephant trumpet calls or other wildlife sound. Paris: Mus'eum national d'Histoire naturelle (2018). https://cran.rproject.org/web/packages/seewave/vignettes/seewave analysis.pdf

12. J.C. Russ. The Image Processing Handbook. Florida: CRC Press (2011). https://www.amazon.com/Image-Processing-Handbook-Sixth/dp/1439840458 